\title{
Síndrome de Burnout: uma análise da saúde mental dos residentes médicos de um Hospital Escola
}

\section{Burnout Syndrome: an analysis of the mental health of medical residents in a teaching hospital}

\author{
José Augusto Costa' (1) drcosta@globo.com \\ Nicoli Abrão Fasanella' (D) nicoli.abrao@gmail.com \\ Beatriz Mendonça Schmitz' (D) biaschmitz03@gmail.com \\ Patrick Cavalcanti Siqueira' (1) siqueirap519@gmail.com
}

\section{RESUMO}

Introdução: A residência médica pode causar a síndrome de burnout, um estado físico, emocional e mental de exaustão extrema.

Objetivo: Buscou-se com esta pesquisa descrever e analisar a prevalência de burnout em médicos residentes vinculados a um hospital-escola e verificar se há correlação com dados sociodemográficos e socioeconômicos.

Método: Este é um estudo analítico, transversal e quantitativo realizado por meio do Maslach Burnout Inventory (MBI) versão Human Services Survey (HSS), do Critério de Classificação Econômica Brasil (CCEB) da Abep e de perguntas sociodemográficas.

Resultado: Dos 221 residentes matriculados, 102 participaram da pesquisa. Destes, 76,47\% apresentaram alto nível em pelo menos um dos três domínios do índice de burnout e $21,57 \%$ dos residentes exibiram alto nível de burnout. Houve relação significativa entre maior número de filhos e presença de exaustão emocional $(p=0,047)$, maior frequência de despersonalização para residentes da área cirúrgica $(p=0,013)$ e reduzida realização profissional com a renda média de $\mathrm{R} \$ 2.965,69$ e $\mathrm{R} \$ 10.386,52$ ( $p=0,006)$. Não foi encontrada relação significativa entre burnout e as variáveis sociodemográficas e socioeconômicas.

Conclusão: Os resultados evidenciam que os médicos residentes estão expostos a situações que contribuem para os altos níveis de estresse e angústia. Ainda são necessários mais estudos sobre o tema.

Palavras-chave: Burnout; Esgotamento Profissional; Corpo Clínico Hospitalar; Médicos Residentes; Depressão.

\begin{abstract}
Introduction: Medical residency can cause burnout syndrome, a physical, emotional and mental state of extreme exhaustion.

Objective: This research sought to describe and analyze the prevalence of burnout in resident physicians linked to a teaching Hospital and to verify whether there is a correlation with sociodemographic and socioeconomic data.

Method: This is an analytical, cross-sectional and quantitative study using the MBI (Malasch Burnout Inventory) version HSS (Human Services Survey), the Brazilian Economic Classification Criterion (CCEB) of ABEP and sociodemographic questions.

Result: Of the enrolled residents, 102 participated in the survey. Of these, $76.47 \%$ showed a high level in at least one of the three domains of the burnout index and $21.57 \%$ of the residents showed a high level of burnout. There was a significant relationship between a larger number of children and the presence of emotional exhaustion $(P=0.047)$, a higher frequency of depersonalization for surgical area residents $(P=0.013)$ and reduced professional accomplishment, with an average income of $R \$ 2,965.69$ and $R \$ 10,386.52(P=0.006)$. No significant relationship was found between burnout and sociodemographic and socioeconomic variables.
\end{abstract}

Conclusion: The results show that resident physicians are exposed to situations that contribute to high levels of stress and distress. Further studies on the subject are still necessary.

Keywords: Burnout; Professional; Medical Staff Hospital; Resident Physicians; Depression.

${ }^{1}$ Pontifícia Universidade Católica de São Paulo, São Paulo, São Paulo, Brasil.

Editora-chefe: Rosiane Viana Zuza Diniz.

Editora associada: Rosiane Viana Zuza Diniz.

Recebido em 05/07/21; Aceito em 09/11/21.

Avaliado pelo processo de double blind review. 


\section{INTRODUÇÃO}

Burnout é um termo da língua inglesa que pode ser entendido como "queima após desgaste"1. Atualmente, é usado para designar um processo de sofrimento psicossocial advindo da situação laboral, uma vez que o ambiente de trabalho e a organização dele podem ser responsáveis pelo sentimento de exaustão física e emocional que causa insatisfação com o trabalho².

Há diversas definições de burnout na literatura. Para Maslach e Jackson ${ }^{3}$, autores consagrados nesse assunto, "Burnout é uma síndrome de exaustão emocional, despersonalização e reduzida realização profissional, que pode ocorrer entre indivíduos que trabalham com pessoas". $\mathrm{Na}$ linha da conceituada pesquisadora norte-americana Christina Maslach, o burnout é caracterizado por três dimensões: exaustão emocional (EE), despersonalização (DE) e reduzida realização profissional (RRP) 4 . A EE ocorre quando o profissional apresenta sentimentos de esgotamento ou falta de energia; a DE acontece como resposta à exaustão, aumentando a distância mental do empregado ou o sentimento de negativismo ou cinismo relacionados ao trabalho de alguém; a RRP se manifesta por conta da falta de recursos pessoais e/ou organizacionais que interferem na redução da eficácia profissional².

Dada a sua relevância, estudos sobre burnout em médicos residentes foram desenvolvidos nos últimos anos. Esses estudos enfatizaram a necessidade de aprofundar o conhecimento sobre o tema e identificar os fatores predisponentes, os riscos e, principalmente, as possíveis mudanças para sua prevenção.

Atualmente, a residência médica é considerada a parte mais desgastante, física e emocionalmente, do processo de especialização profissional do médico ${ }^{5}$. Isso se deve, por um lado, à elevada exigência educacional e, por outro, às requisições do ambiente de trabalho com demandas excessivas que diminuem a qualidade do atendimento, grandes jornadas de trabalho, numerosos plantões, baixa remuneração, necessidade de lidar com sofrimento e morte, exposição constante ao risco, falta de reciprocidade nas relações profissionais e a incerteza quanto ao futuro ${ }^{6}$. Dessa forma, é notória a presença de agentes estressores que propiciam o aparecimento de burnout ${ }^{7}$.

Para fins de comparação, um estudo longitudinal realizado com 422 médicos norte-americanos mostrou que $27 \%$ apresentavam sintomas de burnout ${ }^{8}$. Em outro estudo, foi constatado que mais da metade dos médicos norte-americanos praticantes é afetada pelo burnout ${ }^{2}$. Na China, um estudo transversal envolvendo 1.537 médicos demonstrou que $76,9 \%$ dos entrevistados reportaram algum sintoma e 54,8\% relataram ter cometido algum erro médico ao longo do último ano 9

Quando se observa a situação brasileira, já se sabe que os profissionais da saúde e os professores são as categorias mais investigadas, justamente pelos dados alarmantes provenientes desses estudos ${ }^{2}$. Apesar disso, ainda existe pouca informação sobre burnout no Brasil' ${ }^{2}$ No Rio Grande do Norte, um estudo realizado em três hospitais constatou que $93 \%$ dos profissionais de um desses estabelecimentos apresentavam burnout $^{10}$. Em outro estudo realizado em um hospital público da zona oeste de São Paulo, observou-se que, embora apenas $11 \%$ dos 76 médicos do estudo tenham apresentado burnout, 74\% manifestaram níveis entre moderados e altos de $\mathrm{EE}, 28 \%$ demonstraram RRP e 79\% exibiram índices moderados a altos de $\mathrm{DE}^{11}$. Imersos nesse sofrimento, os residentes médicos ficam mais suscetíveis a transtornos mentais como ansiedade, depressão, abuso de substâncias e ideias suicidas ${ }^{12-14}$. Ao mesmo tempo, as consequências ultrapassam o residente e afetam os pacientes, que podem sofrer com erros médicos evitáveis e também com tratamentos frios e impessoais ${ }^{12,13}$.

A partir desse panorama, esta pesquisa objetivou descrever e analisar a prevalência de burnout em médicos residentes vinculados a um hospital-escola com um programa de residência médica credenciado no Conselho Nacional de Residência Médica desde 1977. Além disso, buscou verificar se há correlação da presença do burnout com dados sociodemográficos e socioeconômicos. Ao fim da pesquisa, foi possível refletir sobre o que isso representa para a formação médica e como é possível contribuir para melhorá-la.

\section{MÉTODO}

\section{Amostra e coleta de dados}

Participaram deste estudo analítico, transversal e quantitativo 102 de 221 residentes médicos pertencentes a todas as áreas oferecidas pelo programa de residência médica e matriculados durante o período de agosto a dezembro de 2019. Os residentes foram convidados a participar da pesquisa pessoalmente e em momentos separados, após reuniões semanais de especialidades. Nessa etapa, eles receberam orientações sobre o tempo de preenchimento do questionário (15 minutos), o caráter anônimo e voluntário da pesquisa, e a assinatura do Termo de Consentimento Livre e Esclarecido (TCLE).

\section{Instrumentos}

A seguir, apresentam-se os instrumentos utilizados nesta pesquisa:

- Os pesquisadores elaboraram um questionário sociodemográfico que avalia as seguintes variáveis: idade, número de filhos, sexo, situação conjugal, ano de residência, especialidade e quantidade de empregos.

- Questionário socioeconômico Critério de Classificação Econômica Brasil (CCEB) da Associação 
Brasileira de Empresas de Pesquisa (Abep): esse instrumento de segmentação econômica é composto por 15 itens e utiliza o levantamento de características domiciliares (presença e quantidade de alguns itens domiciliares de conforto, a origem da água utilizada no domicílio, a presença de asfalto na rua da casa e o grau escolaridade do chefe de família). O CCEB atribui pontos em função de cada característica e realiza a soma deles, de forma a determinar a classe econômica do indivíduo e renda média mensal dele em reais, que será maior, quanto maior for o número de pontos no somatório. Eis a classificação de acordo com a pontuação: 45-100 pontos, classe econômica $A$, com renda média de $\mathrm{R} \$ 25.554,33$; 38-44 pontos, classe econômica B1, com renda média de $\mathrm{R} \$ 11.279,14 ; 29-37$ pontos, classe econômica B2, com R\$ 5.641,64; 23-28 pontos, classe econômica $C 1$, com $\mathrm{R} \$ 3.085,48$; 17 22 pontos, classe econômica $C 2$, com $\mathrm{R} \$ 1.748,59$; e 0-16 pontos, classe D-E, com R\$ 719,81.

- Maslach Burnout Inventory (MBI) versão Human Services Survey (HSS): o MBI é um questionário autoaplicável, com uma escala tipo Likert de cinco pontos, em que se assinalam 0 para nunca, 1 para algumas vezes ao ano, 2 para algumas vezes ao mês, 3 para algumas vezes na semana e 4 para diariamente. $\mathrm{O}$ instrumento, validado no Brasil por Lautert $^{15}$, Tamayo ${ }^{16}$, Benevides-Pereira ${ }^{17}$ e Carlotto et al. $^{18}$, é composto por 22 itens distribuídos em três subescalas: nove questões sobre $E E$, oito sobre realização profissional (RP) e cinco sobre DE. A análise do questionário permite identificar os valores de três variáveis para cada indivíduo, as quais, conforme a pontuação obtida, caracterizam a síndrome de burnout. O preenchimento de apenas um critério não é considerado situação de risco, tampouco ausência de burnout, que se caracteriza por valores acima dos referidos para EE e DE e abaixo do referido para RP.

\section{Análise estatística}

Para a descrição do perfil da amostra, elaboraram-se tabelas de frequência das variáveis categóricas (sexo, situação conjugal, ano de residência, especialidade, realiza outro trabalho) com valores de frequência absoluta ( $n$ ) e percentual (\%), e estatísticas descritivas das variáveis numéricas (idade, renda média, número de filhos), com valores de média, desvio padrão, valores mínimo e máximo, mediana e quartis.

Na comparação das variáveis categóricas da síndrome de burnout, foram utilizados os testes qui-quadrado ou exato de
Fisher. Para a comparação das variáveis numéricas da síndrome de burnout, adotou-se o teste de Mann-Whitney.

Para analisar a relação entre as variáveis numéricas e os escores de burnout, utilizou-se o coeficiente de correlação de Spearman.

O nível de significância adotado para os testes estatísticos foi de $5 \%$, ou seja, $p<0,05$.

Para analisar a consistência interna da escala da síndrome de burnout (MBI), utilizou-se o coeficiente alfa de Cronbach. Valores de alfa acima de 0,60 indicam boa consistência interna. Verificou-se boa consistência interna para os três domínios e o total da escala (alfa $=0,880$ ).

$\mathrm{Na}$ análise estatística, adotou-se o Statistical Analysis System (SAS) versão 9.2.

\section{RESULTADOS}

O número total de entrevistados foi de 102 residentes médicos, dentro de um grupo de 221 residentes médicos matriculados no ano de 2019. O grupo de respondentes apresentou uma média simples de idade de 28,18 anos. Houve maior frequência do sexo feminino $(60,78 \%)$, de solteiros $(83,33 \%)$, sem filhos $(96,08 \%)$, com a realização de outro trabalho $(69,61 \%)$ e renda média de $\mathrm{R} \$ 5.363,19$ mensais $(50,98 \%)$.

A maior parte dos residentes era da área clínica $(27,45 \%)$ e da área cirúrgica (22,55\%). Residentes da ortopedia corresponderam a $15,69 \%$ da amostra, acompanhados pelos residentes da pediatria (13,73\%), ginecologia e obstetrícia $(11,76 \%)$, psiquiatria $(4,90 \%)$ e outros $(3,92 \%)$.

Para o diagnóstico de síndrome de burnout, consideraram-se valores acima da média para EE e DE e abaixo da média para RP. As médias das subescalas estão apresentadas na Tabela 1.

A Tabela 2 apresenta os níveis de EE, DE e RP: 50,98\% da amostra apresentou alto nível de $\mathrm{EE}, 50 \%$ exibiram alto nível de DP e 42,16\% tiveram um nível baixo de RP. Na amostra, 21,57\% apresentaram alto nível de burnout, e 76,74\%, alto nível em pelo menos uma das três dimensões avaliadas.

A Tabela 3 representa a porcentagem do alto nível de cada componente de burnout distribuído de acordo com as

Tabela 1. Distribuição dos residentes médicos segundo as médias do Maslach Burnout Inventory.

\begin{tabular}{lcccc}
\hline \multicolumn{1}{c}{ Subescalas } & Média & DP* & Mín. & Máx. \\
Exaustão emocional & 20,78 & 7,22 & 3 & 36 \\
Despersonalização & 6,52 & 3,75 & 0 & 15 \\
Realização profissional & 21,62 & 4,76 & 8 & 32 \\
\hline
\end{tabular}

*DP: Desvio padrão.

Fonte: Elaborada pelos autores. 
Tabela 2. Dimensões dos residentes médicos quanto às dimensões isoladas do Maslach Burnout Inventory e de burnout.

\begin{tabular}{lcccccc}
\hline & \multicolumn{2}{c}{ EE } & \multicolumn{2}{c}{ DE } & \multicolumn{2}{c}{ RP } \\
\cline { 2 - 7 } & $\%$ & Frequência & $\%$ & Frequência & $\%$ & Frequência \\
\hline Nível baixo & 49,02 & 50 & 50 & 51 & 42,16 & 43 \\
Nível alto & 50,98 & 52 & 50 & 51 & 57,84 & 59 \\
\hline \multicolumn{2}{l}{} & & & & $\%$ & Frequência \\
\hline Síndrome de burnout & & & & 21,57 & 22 \\
Alto nível em pelo menos um domínio do índice &
\end{tabular}

Fonte: Elaborada pelos autores.

Tabela 3. Relação entre frequência de burnout e cada uma de suas dimensões com características sócio-ocupacionais da amostra.

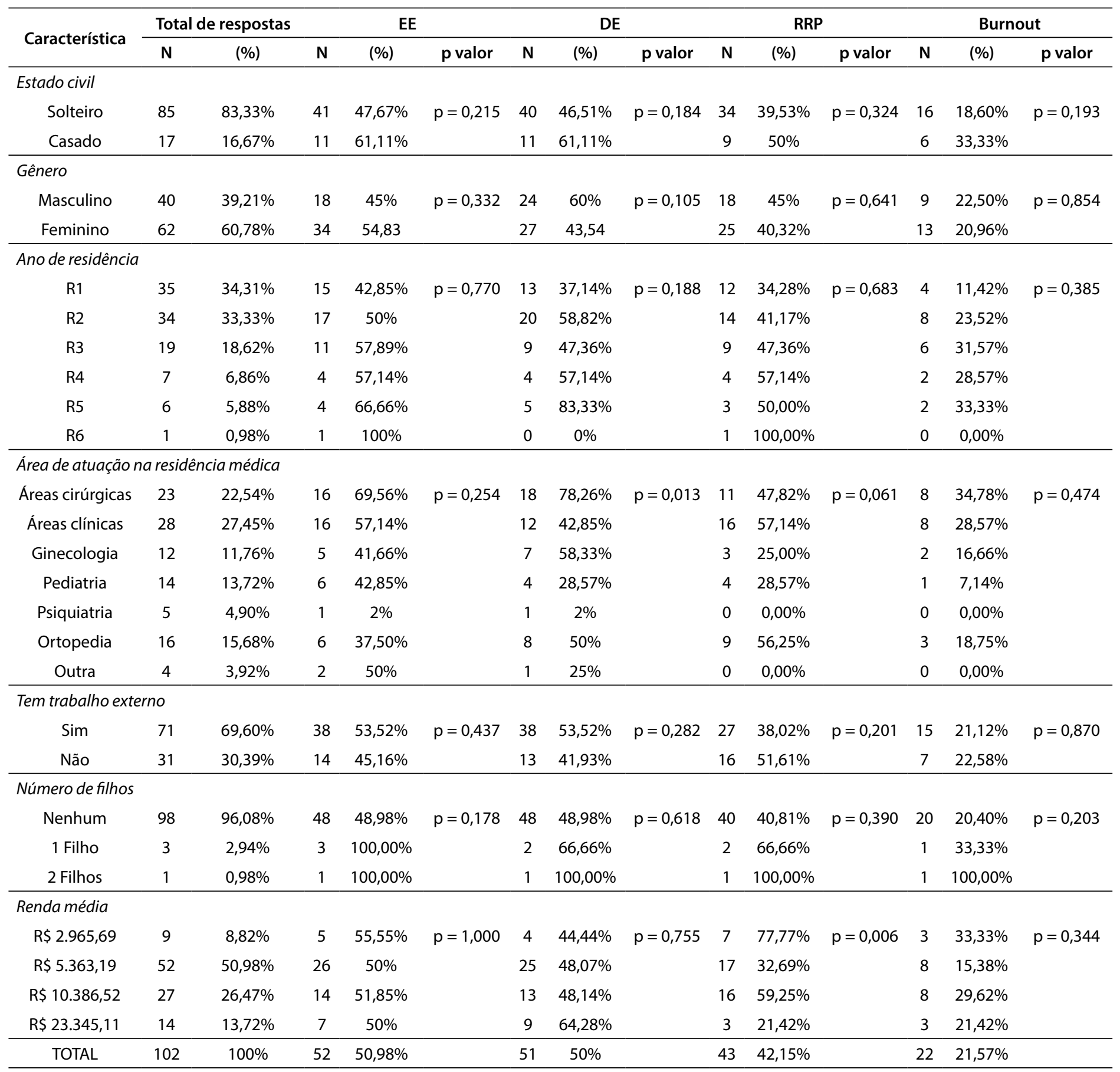

Fonte: Elaborada pelos autores. 
diferentes variáveis demográficas da pesquisa e os resultados dos testes estatísticos inferenciais.

Dos 22 entrevistados que apresentaram valores acima da média para EE e DE e abaixo da média para RP caracterizando a síndrome, 13 eram do sexo feminino e nove do masculino. Em percentual, esses valores significam que $22,5 \%$ dos homens e $20,96 \%$ das mulheres apresentaram indicadores de burnout. Os homens apresentaram níveis mais altos de DE (60\%) e níveis mais baixos de RP (45\%) do que as mulheres $(43,54 \%)$ e $(40,32 \%)$, respectivamente. As mulheres mostraram níveis mais altos de EE $(54,83 \%)$ do que os homens (45\%). Porém, não foram encontradas relações significativas entre essas variáveis.

Não se encontraram diferenças estatisticamente significativas na relação entre o estado conjugal dos entrevistados e ter burnout ou com a frequência de qualquer uma de suas subescalas EE, DE e RRP. Também não se constataram diferenças da estratificação da amostra por faixa de renda, com exceção de $p$ alfa significativo para a dimensão de RRP naqueles que declararam renda média mensal de R\$ $2.965,00$ e $R \$ 10.386,00$ ( $p=0,006)$.

Pelos resultados, verificou-se uma diferença de associação entre EE e número de filhos. Quanto maior o número de filhos, maior o valor da EE $(p=0,047)$.

Os residentes que trabalham fora apresentaram as maiores taxas de $\operatorname{EE}(53,52 \%)$ e $\mathrm{DE}(53,52 \%)$, enquanto os residentes que não realizam outro trabalho apresentaram menores valores de RP $(51,62 \%)$.

Os médicos do quinto ano de residência apresentaram o maior percentual de EE, DE e síndrome de burnout, porém não foi encontrada relação significativa entre essas variáveis. Além disso, o Gráfico 1 demonstra que os níveis gerais de esgotamento tendem a ser mais altos à medida que os anos de residência aumentam.

Na comparação entre especialidades, não foi encontrada relação significativa entre essa variável e os níveis de burnout, embora os residentes da área cirúrgica tenham o maior nível de $\operatorname{DE}(78,26 \%)(p=0,013)$.

Gráfico 1.Níveis gerais de esgotamento relacionado com os anos de residência.

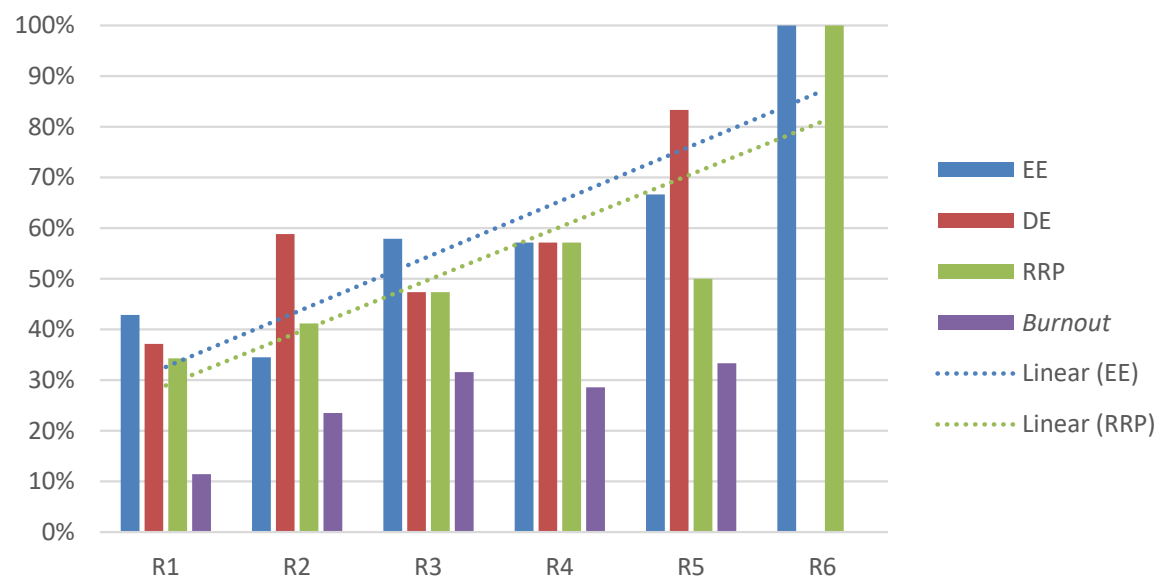

Fonte: Elaborado pelos autores.

Gráfico 2.Relação entre as três dimensões do burnout.

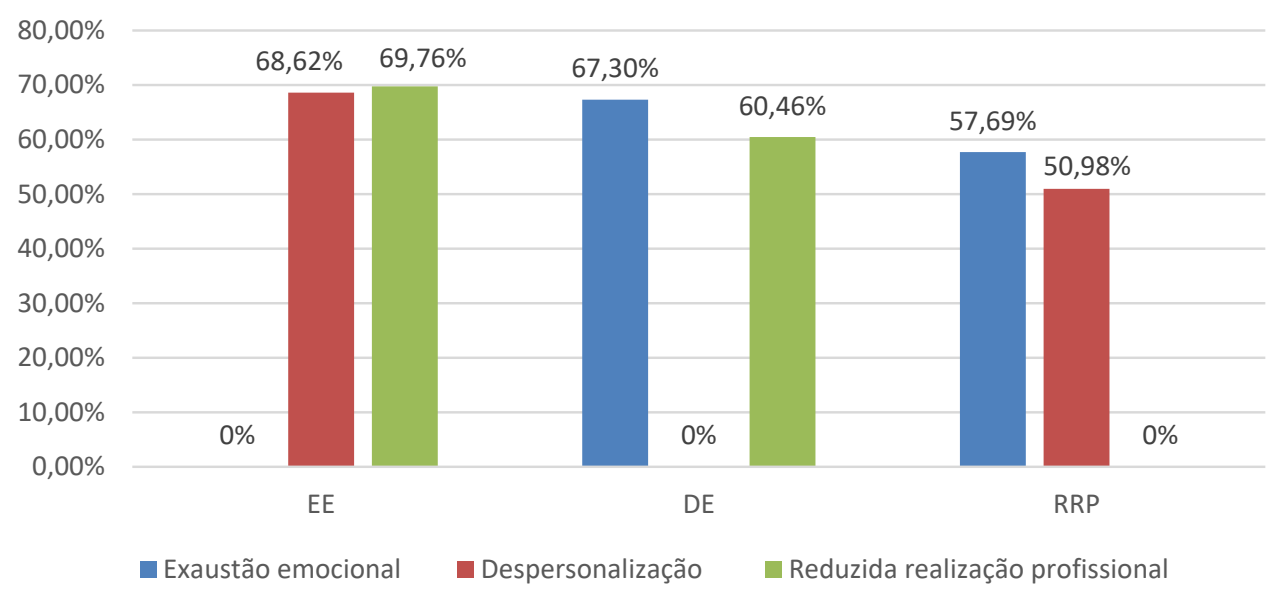

Fonte: Elaborado pelos autores. 
Pelos resultados, verificou-se diferença significativa entre $E E$ para $D E(p<0,001)$ e RRP $(p=0,001)$ (maior frequência de sim nos com $E E)$, escore $D E(p<0,001)$ (maiores valores nos com EE) e escore RRP ( $p<0,001$ ) (menores valores nos com EE). Verificou-se também diferença significativa entre DE para EE ( $p$ $<0,001$ ) (maior frequência de sim nos com DE) e escore EE ( $p<$ 0,001 ) (maiores valores nos com DE). Outrossim, houve $p$ alfa significativo entre RRP para $E E(p=0,001)$ (maior frequência de sim nos com RRP) e escores EE $(p<0,001)$ e DE $(p=0,005)$ (maiores valores nos com RRP) - ver Gráfico 2.

\section{DISCUSSÃO}

A residência médica no Brasil, almejada pela maioria dos estudantes de Medicina, é vista como a validação de um sonho e a chance de progressão na carreira. Entretanto, os desafios que esse momento impõe acabam afetando aqueles que se propõem a realizar uma especialização visando à realização pessoal, profissional e financeira. Conhecida como o período mais árduo da vida de um médico, a residência é cercada por altas cobranças, longas horas de trabalho, baixa remuneração e uma dualidade entre ser médico e ainda viver e ser tratado como um estudante $e^{5-7,19}$. Diante disso, a alta suscetibilidade desses profissionais ao burnout é o que justifica este estudo e muitos outros no mundo todo.

Os sintomas de burnout na medicina podem se originar de várias causas, como requisitos burocráticos, mudança contínua dos ambientes de trabalho, má administração hospitalar, supervisão clínica precária, relatórios sensacionalistas da mídia sobre erros médicos, recursos limitados de saúde e baixo equilíbrio entre trabalho e vida pessoal20,21.

Em nosso estudo, a prevalência de síndrome de burnout foi de 21,56\%, com 102 respondentes. De acordo com o Conselho Federal de Medicina (CFM), no Brasil 23,1\% dos médicos apresentam síndrome de burnout em alto grau ${ }^{22}$. Em outras publicações nacionais, a presença dessa síndrome varia de $20,8 \%$ a $81,5 \% \%^{1,19}$. Em estudo de metanálise mundial com 22.778 indivíduos, identificou-se uma prevalência agregada de burnout de $51 \%{ }^{21}$, enquanto, em um estudo sírio, a prevalência de burnout em 3.350 residentes foi de $19,3 \%{ }^{23}$.

Dessa forma, infere-se que, apesar de o nosso estudo apresentar resultados semelhantes com a média nacional do CFM, ao comparar com outros estudo nacionais e internacionais, existe muita discrepância e variação, de aproximadamente $60 \%$, nos resultados. Essa diferença decorre das variáveis pontuações que definem a existência de burnout na literatura. Em uma publicação inglesa, a presença da síndrome é definida pela alteração nas três dimensões ( $D E$, EE e RRP) ${ }^{24}$, mas, em um estudo canadense, o burnout é definido pela presença de alteração em apenas uma das três dimensões ${ }^{25}$. Nos estudos semelhantes ao nosso, feitos em hospitais universitários com residentes médicos ${ }^{1,19,23}$, o critério para a definição de burnout foi a presença das três dimensões, e, caso haja a presença de apenas duas, a síndrome é considerada como situação de risco, da mesma forma definida pela nossa pesquisa. Diante disso, é necessário considerar se a pesquisa precisa aumentar a amostra estudada, buscando um intervalo de confiança maior, ou se o instrumento do $\mathrm{MBI}$ ainda é adequado, visto que ele é datado da década de $1980^{3,22}$, de forma a atualizar e padronizar qual seria a definição ideal da presença ou ausência da doença.

A amostra de residentes médicos estudados na pesquisa apresentou maioria dos integrantes do sexo feminino $(60,78 \%)$, dos quais 13 apresentaram burnout, representando $20,96 \%$ do total de mulheres. Já os homens foram apenas $39,21 \%$ da amostra, mas apresentaram 22,5\% de burnout na amostra estudada. Sobre o total de entrevistados com critérios para burnout, que somaram 22 indivíduos, 59,09\% eram mulheres, enquanto 40,9\% eram homens. Dessa forma, o cenário de maioria feminina na amostra nos centros de ensino médico pelo mundo se repete em outros estudos, com números entre $56,30 \%$ e $69,3 \%$ de mulheres no Canadá ${ }^{12,26}$, e, em Porto Alegre, com 58,3\% de mulheres, o que evidencia uma mudança sociodemográfica de mais de 60 anos, com aumento das profissionais médicas, que na década de 1970 eram de $23,47 \%{ }^{27}$ e em 2018 foram de $45,6 \%$ dos profissionais formados ${ }^{28}$, apresentando uma expectativa de ser maioria no mercado de trabalho médico em $2028^{27}$. Entretanto, apesar de o sexo feminino aparecer como maioria absoluta, o sexo masculino ainda é o mais afetado pelo burnout, como indicam estudos nacionais e internacionais ${ }^{19,21,23}$. Isso evidencia, portanto, que os homens, possivelmente, sofrem mais com os fatores estressantes da vida médica, como baixo valor da bolsa de residência, alta cobrança de chefes e pacientes, ter muitas tarefas administrativas, além de trabalhar longas horas, falta de respeito e compensação insuficiente, que são somados ao papel atribuído socialmente ao homem de provedor da casa e de ser bem-sucedido financeiramente ${ }^{23,29}$. Apesar disso, a associação entre sexo e prevalência de burnout não é bem consolidada e mostra discrepâncias em outros estudos, o que indica a possibilidade de não ser um bom indicador demográfico para prevalência da síndrome $e^{1,19,30}$.

Quando se observam as dimensões da síndrome no estudo, as mulheres são maioria na EE (65,38\%), na DE (52,94\%) e na $\operatorname{RRP}(58,14 \%)$. Entretanto, em outros estudo nacionais, o sexo feminino lidera a $E E$, enquanto o masculino lidera a $\mathrm{DE}^{1,19}$, mas, segundo o estudo sírio, os homens lideram as três dimensões ${ }^{23}$. Os resultados nacionais aparecem em consonância a uma metanálise internacional sobre burnout e gênero, que indica uma maior prevalência de EE referida por mulheres, 
enquanto os homens referem mais $\mathrm{DE}^{30}$. Apesar disso, o fato de ser voluntária a aplicação do questionário, de a amostra ser predominantemente feminina e de as relações entre sexo e presença da síndrome sofrerem bastante discrepância na literatura mundial, não é possível afirmar uma relação direta entre algum dos espectros e sexo.

Quanto ao estado civil do grupo em estudo, o fato de ser casado mostrou ser um possível fator de risco, já que $35,29 \%$ dos participantes casados apresentaram burnout contra $18,82 \%$ dos solteiros; além disso, aqueles apresentaram maiores índices de DE, EE e RRP. Esse dado consta em outras publicações nacionais, em que os residentes casados aparecem com maior risco de desenvolver a síndrome ${ }^{1,19}$. Entretanto, quando se analisaram os espectros nesses estudos, os solteiros apresentaram maiores valores de DE e RRP ${ }^{1}$. Contudo, um outro estudo $^{19}$ indica resultados diferentes. Dessa forma, é possível inferir que a necessidade de equilibrar as demandas familiares e de relacionamento afetivos com grandes cargas de responsabilidade afetiva pode ser um fator estressor associado aos fatores inerentes à atividade do residente médico, mas sem excluir a possibilidade de os solteiros apresentarem fatores de risco para o desenvolvimento de burnout também.

Sobre o número de filhos, não tê-los se mostrou um possível fator protetor, uma vez que, nesse grupo, apenas 20,4\% apresentaram conjunto de dimensões que caracterizam burnout. Esse aspecto mostrou-se como um possível fator de risco para ter EE e DE, mas sem relação com RP. Isso pode ser devido ao fato de os indivíduos sem filhos não carregarem mais preocupações ou responsabilidades que podem ser fatores desencadeadores de estresse, como família e filhos, associados aos fatores estressantes da atuação médica. Entretanto, não foram encontrados outros estudos para comparação.

$\mathrm{O}$ ano de residência demonstrou ser um possível fator de risco, ou seja, é provável um aumento de risco de desenvolver a síndrome conforme mais anos de residência completos, sendo de $11,4 \%$ no residente de primeiro ano e $33 \%$ no residente de quinto ano. Esse resultado é similar a outro estudo nacional ${ }^{19}$ e ao estudo sírio ${ }^{23}$, apesar de nenhum dos três estudos apresentar associação significativa entre ano de residência e presença da síndrome. Essa tendência pode indicar uma mudança de expectativas desse residente com a profissão conforme o avanço da residência ou um aumento de sonhos e responsabilidades à medida que se aproxima o fim da residência e aumenta a responsabilidade pelo paciente e pelo próprio futuro profissional. Além disso, são altas a expectativa e a energia no início do programa, as quais podem ser perdidas e dar lugar aos sintomas de burnout conforme o tempo passa. Ademais, a EE aumenta de incidência conforme aumenta o tempo de residência, com $42,85 \%$ no primeiro ano e $57,89 \%$ no terceiro ano, como também pode ser observado no estudo de Porto Alegre e no estudo sírio ${ }^{19,23}$, ainda assim sem relevância significativa entre síndrome e ano de residência. Observa-se também um provável aumento da redução da RP à medida que avança no ano de residência, de forma contrária aos estudos de Porto Alegre e sírio ${ }^{19,23}$, o que pode indicar uma mudança de expectativa profissional ou desrealização com a escolha feita, sendo de $34,28 \%$ no primeiro ano e de $47,36 \%$ no terceiro ano.

No que concerne à variável das especialidades, a maior parte dos participantes foi das áreas conhecidas como prérequisito para a realização de outra residência, como a clínica médica $(22,55 \%)$ e a cirúrgica $(27,45 \%)$. Quando se observa a presença de espectros e de burnout, a área cirúrgica aparece como um possível fator de risco para o desenvolvimento da síndrome $(34,78 \%)$, seguida pela clínica $(28,57 \%)$, o que está em consonância com a metanálise internacional que indicou não haver diferença da incidência de burnout nas duas áreas ${ }^{21}$. Apesar disso, não foram encontrados estudos com correlação significativa entre especialidade e maior presença de burnout. Dessa forma, pode-se inferir uma provável relação entre as áreas que se configuram como pré-requisito com um aumento de risco para a doença, de forma que é possível questionar se o prolongamento da formação do profissional médico, que se vê obrigado a fazer duas residências para então poder atuar na especialidade de sua escolha, pode vir a ser um fator que agride a sua saúde física e mental e levanta o questionamento sobre esse modelo de formação. A especialidade escolhida, entretanto, não parece afetar $\mathrm{EE}$, mas a área cirúrgica apresenta maior taxa de EE com $60,86 \%$. Ela aparece também como fator de risco $(78,26 \%)$ para o desenvolvimento da $\mathrm{DE}$, enquanto a área clínica $(42,85 \%)$ e a pediatria $(28,57 \%)$ aparecem como protetoras ( $p=$ 0,013). Nos estudos sírio e mineiro, os espectros não mostraram estar mais presentes em determinadas áreas, não indicando associação ${ }^{1,23}$. Apesar disso, no estudo gaúcho, as áreas cirúrgica e de pediatria apareceram como possíveis fatores protetores para exaustão profissional e $\mathrm{DE}^{19}$. A escolha da especialidade não parece influenciar na RP, indicando, provavelmente, que a área não é importante para definir esse sentimento.

Quanto à renda, ela parece não influenciar significativamente na presença ou não da síndrome, apesar de aqueles com menor renda $(\mathrm{R} \$ 2.965,69)$ terem apresentado o maior valor de burnout (33,33\%), consoante a outro estudo brasileiro ${ }^{19}$, em que $85,5 \%$ dos indivíduos com renda menor que R\$3.000,00 apresentaram burnout. Entretanto, nenhuma publicação foi encontrada com associação significativa entre renda média mensal e presença da doença em estudo. Dessa forma, a baixa remuneração pode parecer um fator de risco, mas sem evidência, devido ao fato de a realidade financeira ser relativa às necessidades de cada indivíduo e da possibilidade 
de realizar outros trabalhos além da residência. Observando os espectros, em nosso estudo a renda média mostrou associação significativa em relação à $\mathrm{RRP}$, mas com variações que não permitem afirmar se mais ou menos renda significaria maior ou menor $\operatorname{RP}(p=0,006)$, de maneira semelhante ao estudo de Porto Alegre, que mostrou variação da RP entre os valores de renda, mas sem associação significativa ${ }^{19}$, de forma que traz a reflexão sobre o quanto o dinheiro, que é algo objetivo, pode influenciar no sentimento de RP, que é algo subjetivo.

Na pesquisa, $69,60 \%$ dos entrevistados realizavam outro trabalho além da residência. Entretanto, realizar outro trabalho não mostrou indicação para um possível risco ou proteção para o desenvolvimento da síndrome. Em outra publicação nacional, também não houve associação significativa entre burnout e realização de outro trabalho ${ }^{19}$. Em um estudo internacional, o espectro de EE foi correlacionado com o alto número de horas trabalhada ${ }^{31}$, enquanto, no nosso estudo, aqueles que trabalham além da residência, e portanto mais horas, não apresentaram mais EE do que aqueles que realizam apenas as horas de trabalho previstas no programa de residência. Além disso, a realização de outro trabalho apareceu como possível fator protetor em relação à redução da $\operatorname{RP}(38,02 \%)$, de forma semelhante ao estudo de Porto Alegre ${ }^{19}$. Dessa forma, entende-se que realizar outro trabalho pode proporcionar mais gratificação ao residente que, além de ser aluno, pode assumir a posição de médico em outro momento, o que resultará em maior satisfação profissional.

Quanto à distribuição dos entrevistados nas três dimensões que avaliam o burnout, os valores são praticamente os mesmos: $50,98 \%$ para EE e $50 \%$ para DE. Entretanto, no caso da reduzida $\mathrm{RP}, 57,84 \%$ se dizem realizados profissionalmente. Para Tamayo ${ }^{16,32}$, a EE é a dimensão aceita com facilidade pelo profissional, o que permite com que ele entenda, expresse e admita mais facilmente essa sensação. Em outros estudos nacionais, os resultados são parecidos variando de $53 \%$ a $65 \%$ em $E E$, de $47,7 \%$ a $61,7 \%$ em DE e de $30 \%$ a $45 \%$ em RRP ${ }^{1,19}$, de forma semelhante ao estudo sírio com $77,9 \%$ de $E E, 54,6 \%$ de DE e 13,7\% de RRP. Portanto, é possível inferir que, apesar da alta em EE e DE, a RP consegue ser sentida pela maioria dos residentes, mesmo com estresse físico e mental, baixa remuneração e longas horas de trabalho. Dessa forma, apesar de os outros dois fatores que demonstram o início de uma redução da qualidade de vida estarem presentes na maioria dos entrevistados, eles ainda se sentem realizados, o que condiz com a definição de reduzida $\mathrm{RP}$ de Marshal, que a relaciona à estrutura oferecida e à possibilidade de realizar o seu trabalho, o que parece ser positivo nos programas de residência do país e do mundo, inclusive no hospital-escola estudado. Além disso, é necessário pontuar que, devido à valorização dentro da sociedade médica de escolas tradicionais, que a maior RP observada no estudo pode em parte ser atribuída ao fato de que o hospital-escola estudado oferece especialização médica desde 1977, sendo reconhecido no mercado médico.

Sobre a presença de EE no cenário de DE e vice-versa, o resultado da nossa pesquisa demonstra uma relação direta entre essas variáveis. Esse resultado é consoante ao estudo de Curitiba que entrevistou 136 residentes e constatou alto nível de EE e DE com moderado nível de RP, assim como outros estudos nacionais de Porto Alegre e Uberlândia, além de estudos internacionais da Síria e do Canadá, que apresentaram resultados semelhantes ${ }^{1,5,19,23,26}$. Dessa forma, é possível concluir que $\mathrm{DE}$ e $\mathrm{EE}$, nesta pesquisa, acontecem simultaneamente, o que indica qual indivíduo apresenta alto risco para o desenvolvimento da doença, sendo a RP determinante na classificação ou não de cada participante dentro da síndrome de burnout, evidenciando aqueles que devem ser alvos de intervenções.

Este estudo apresenta algumas limitações que merecem ser citadas. Em primeiro lugar, em decorrência da dificuldade de acesso aos residentes e da incompatibilidade de horários com os pesquisadores, alguns grupos não conseguiram participar da pesquisa. Além disso, durante as abordagens em reuniões clínicas, alguns residentes estavam ausentes e, por isso, não foram incluídos na pesquisa. Além disso, alguns dos participantes já tinham conhecimento sobre o tema, o que pode ter ocasionado um viés por familiaridade ou pelo próprio sofrimento psíquico e estresse. Por fim, alguns residentes se mostraram receosos em responder, por medo de que as informações contidas, principalmente sobre o trabalho externo, chegassem ao conhecimento de seus chefes, uma vez que a residência é um programa de alta dedicação. Dessa forma, a amostra pode ter sofrido algum viés de seleção. Em segundo lugar, a pesquisa aborda questões subjetivas que, apesar de validadas e consagradas no MBI-HSS, podem ser consideradas viés de aferição. Em terceiro lugar, por ser um estudo transversal, não permite inferir causalidade, necessitando de mais estudos. Por fim, a amostra de 102 residentes não representa a totalidade de residentes da instituição, o que mostra a necessidade de ampliar o estudo para melhores resultados.

Com base no que foi exposto neste estudo, é possível inferir que, apesar não ser um grande número de residentes com todos os espectros e critérios diagnósticos para síndrome de burnout, a maioria dos participantes da pesquisa apresentou ao menos um aspecto entre EE, DE e RRP, sendo aproximadamente metade deles com EE e DE. Diante disso, deve-se pontuar que eles se apresentam em algum nível de sofrimento e que intervenções são necessárias para evitar o agravamento da situação. Dessa forma, podemos sugerir uma 
melhor remuneração, para que a questão financeira não seja um possível fator de sofrimento, além de permitir a sensação de reconhecimento pelo trabalho e pelas horas semanais dedicadas. Isso evitaria também a necessidade de realizar outro trabalho, permitindo mais tempo livre para investir no próprio indivíduo realizando atividades que proporcionem relaxamento e melhorem o bem-estar e a saúde física e mental. Ademais, a instituição deve liderar grupos de apoio, não obrigatórios, com profissionais capacitados para oferecer apoio psíquico aos que desejarem, uma vez que na instituição pesquisada havia apenas um canal de apoio para toda a comunidade da faculdade (graduandos, professores, funcionários e residentes), e não específica para residentes médicos e suas particularidades. É necessário rever também as peculiaridades das especialidades, visto que repetidamente a que apresentou a maior chance de risco e de sofrimento, neste estudo, foi a área cirúrgica, o que pode sugerir a existência de características que podem colocar em risco os residentes que vivenciam essa especialização. Além disso, quando os residentes médicos têm a saúde física e a saúde mental prejudicadas, isso afeta indiretamente a qualidade de tratamento destinado aos pacientes ${ }^{33}$ e aumenta o risco de erros evitáveis ${ }^{9,12,13}$. As evidências mostram erros de prescrição e redução da qualidade dos serviços médicos devido à exaustão, afetando potencialmente o relacionamento interprofissional ${ }^{21,33}$. Como estratégia de prevenção, os estudos mostram que três mudanças são recomendadas para reduzir o risco de burnout: a modificação da estrutura organizacional, a adequação entre a organização e o médico, e ações em nível individual, por meio de comportamentos saudáveis ${ }^{21,34}$. Portanto, são necessários mais estudos sobre o tema, a fim de compreender de forma mais eficaz o atual cenário e os agravantes dele, e indicar as melhores formas de intervenção para diminuir o esgotamento físico e psíquico dos residentes médicos e aprimorar a qualidade de atendimento.

\section{CONCLUSÃO}

A prevalência de burnout entre os médicos residentes foi de $21,57 \%$, consistente com a literatura. Isso pode indicar que os médicos residentes estão sendo expostos a uma infinidade de emoções que contribuem para os altos níveis de estresse e angústia.

Além disso, este estudo mostrou que houve uma relação significativa entre residentes da área cirúrgica com a $D E$, maiores valores de EE quanto maior o número de filhos e uma relação direta entre a presença de EE, $D E$ e redução da $\mathrm{RP}$.

Por fim, são necessários mais estudos para entender os mecanismos de enfrentamento, a fim de prevenir e reduzir os efeitos do burnout nos residentes médicos. Deve-se criar um ambiente no qual os residentes prosperem, alcancem bem-estar e adquiram resiliência. A conquista desses objetivos ajudará a promover a competência, a dedicação e o profissionalismo dos futuros médicos, durante o processo de treinamento e ao longo de suas carreiras.

\section{AGRADECIMENTOS}

Os autores agradecem ao estatístico Helymar Machado a realização das análises e o gerenciamento do banco de dados.

\section{CONTRIBUIÇÃO DOS AUTORES}

José Augusto Costa participou da pesquisa bibliográfica, da análise dos dados e da revisão crítica. Nicoli Abrão Fasanella participou da pesquisa bibliográfica, da coleta e análise dos dados, e da redação final do texto. Beatriz Mendonça Schmitz e Patrick Cavalcanti Siqueira participaram da pesquisa bibliográfica, da coleta dos dados, da análise dos dados e da redação final do texto.

\section{CONFLITO DE INTERESSES}

Declaramos não haver conflito de interesses.

\section{FINANCIAMENTO}

Este estudo foi financiado pelo Conselho de Ensino, Pesquisa e Extensão (Cepe) da Pontifícia Universidade Católica de São Paulo (PUC-SP).

\section{REFERÊNCIAS}

1. Lima FD, Buunk AP, Bernadete $M$, Araújo J, Gomide J, Chaves $M$, et al. Síndrome de burnout em residentes da Universidade Federal de Uberlândia - 2004. Rev Bras Educ Med. 2007;31(2):137-46.

2. Mendanha M, Bernardes $P$, Shiozawa P. Desvendando o burnout: uma análise interdisciplinar da síndrome do esgotamento profissional. São Paulo: LTr; 2018.

3. Maslach C, Jackson SE. The measurement of experienced burnout. J Organ Behav. 1981;2(2):99-113.

4. Soares LR, Lopes TMO, Silva MAO, Ribeiro MVA, Almeida Júnior MP, Silva RA, et al. Burnout e pensamentos suicidas em médicos residentes de hospital universitário. Rev Bras Educ Med. 2012;36(1):77-82.

5. Asaiag PE, Perotta B, Martins MA, Tempski P. Avaliação da qualidade de vida, sonolência diurna e burnout em médicos residentes. Rev Bras Educ Med. 2010;34(3):422-9.

6. Lockley SW, Cronin J, Evans EE, Cade BE, Clark JL, Landrigan C, et al. Effect of reducing interns' weekly work hours on sleep and attentional failures. $\mathrm{N}$ Engl J Med. 2004;351(15):1493-501.

7. Madsen T. Resident burnout. JAMA. 2004;292(23):2880-9.

8. Linzer M, Manwell LB, Mundt M, Williams E, Maguire A, McMurray J, et al. Organizational climate, stress, and error in primary care: the MEMO study. Adv Patient Saf. 2005;1:65-78.

9. Wen J, Cheng Y, Hu X, Yuan P, Hao T, Shi Y. Workload, burnout, and medical mistakes among physicians in China: a cross-sectional study. Biosci Trends. 2016;10(1):27-33.

10. Borges L, Argolo J, Pereira A, Machado E, Silva W. A Síndrome de burnout e os valores organizacionais: um estudo comparativo em hospitais universitários. Psicol Reflex Crit. 2002;15(1):189-200. 
11. Magalhães RAC, Glina DMR. Prevalência de burnout em médicos de um hospital público de São Paulo. Saúde, Ética \& Justiça. 2006;11(1-2):29-35.

12. Kealy D, Halli P, Ogrodniczuk JS, Hadjipavlou G. Burnout among Canadian psychiatry residents: a national survey. Can J Psychiatry. 2016;61(11):732-6.

13. Rodrigues $\mathrm{H}$, Cobucci R, Oliveira A, Cabral JV, Medeiros L, Gurgel K, et al. Burnout syndrome among medical residents: a systematic review and meta-analysis. PLoS One. 2018;13(11):1-17.

14. West CP, Dyrbye LN, Erwin PJ, Shanafelt TD. Interventions to prevent and reduce physician burnout: a systematic review and meta-analysis. Lancet. 2016;388(10057):2272-81.

15. Lautert L. O desgaste profissional do enfermeiro [dissertação]. Salamanca: Universidad Pontificia de Salamanca; 1995.

16. Tamayo MR. Relação entre a síndrome de burnout e os valores organizacionais no pessoal de enfermagem de dois serviços públicos [dissertação]. Brasília: Universidade de Brasília; 1997.

17. Benevides-Pereira AMT. MBI - Maslach Burnout Inventory e suas adaptações para o Brasil. Anais da Reunião Anual de Psicologia. Rio de Janeiro: Sociedade Brasileira de Psicologia; 2001.

18. Carlotto MS, Câmara SG. Propriedades psicométricas do Maslach Burnout Inventory em uma amostra multifuncional. Estud Psicol. 2007;24(3):325-32.

19. Bond MMK, de Oliveira MS, Bressan BJ, Bond MMK, da Silva ALFA, Merlo ÁRC. Prevalência de burnout entre médicos residentes de um hospital universitário. Rev Bras Educ Med. 2018;42(3):97-107.

20. Kumar S. Burnout and doctors: prevalence, prevention and intervention. Healthcare. 2016;4(37):1-9.

21. Low ZX, Yeo KA, Sharma VK, Leung GK, Mclntyre RS, Guerrero A, et al. Prevalence of burnout in medical and surgical residents: a meta-analysis. Int J Environ Res Public Health. 2019;16(9):1-22.

22. Moreira HA, de Souza KN, Yamaguchi MU. Síndrome de burnout em médicos: uma revisão sistemática. Rev Bras Saúde Ocup. 2018;43(3):1-11.

23. Alhaffar BA, Abbas $G$, Alhaffar AA. The prevalence of burnout syndrome among resident physicians in Syria. J Occup Med Toxicol. 2019;14(31):1-8.
24. Ramirez AJ, Graham J, Richards MA, Cull A, Gregory WM, Leaning MS, et al. Burnout and psychiatric disorder among cancer clinicians. Br J Cancer. 1995;71(6):1263-9.

25. Grunfeld E, Whelan TJ, Zitzelsberger L, Willan AR, Montesanto B, Evans WK. Cancer care workers in Ontario: prevalence of burnout, job stress and job satisfaction. Can Med Assoc J. 2000;163(2):166-9.

26. Ferguson C, Low G, Shiau G. Resident physician burnout: insights from a Canadian multispecialty survey. Postgrad Med J. 2020;0:1-8.

27. Scheffer M., coordenador. Demografia médica no Brasil. São Paulo: Cremesp; 2013.v. 2.

28. Associação Médica Brasileira. Demografia médica 2018: o perfil do médico brasileiro e a desigualdade no acesso à assistência. Rev Med (Puebla). 2018;1(4):1-35.

29. Mannam S. Are physicians happy with their jobs ? Addressing physician burnout. J Young Investig. 2019;37(1):6-7.

30. Purvanova RK, Muros JP. Gender differences in burnout: a meta-analysis. J Vocat Behav. 2010;77(2):168-85.

31. Gabbe SG, Melville J, Mandel L, Walker E. Burnout in chairs of obstetrics and gynecology: diagnosis, treatment, and prevention. Am J Obstet Gynecol. 2002;186(4):601-12.

32. Tamayo MR. Burnout: implicações das fontes organizacionais de desajuste indivíduo-trabalho em profissionais da enfermagem. Psicol Reflex e Crit. 2009;22(3):474-82.

33. Dewa $C S$, Loong $D$, Bonato $S$, Trojanowski L, Rea M. The relationship between resident burnout and safety-related and acceptability-related quality of healthcare: a systematic literature review. BMC Med Educ. 2017;17(195):1-16.

34. Lee RT, Seo B, Hladkyj S, Lovell BL, Schwartzmann L. Correlates of physician burnout across regions and specialties: a meta-analysis. Hum Resour Health. 2013;11(48):1-16. 